\title{
the Unione Matematica Italiana
}




\section{Editorial Board}

Ciro Ciliberto (Editor in Chief)

Dipartimento di Matematica

Universita' di Roma Tor Vergata

Via della Ricerca Scientifica

00133 Roma (Italia)

e-mail: cilibert@axp.mat.uniroma2.it

Susanna Terracini (Co-editor in Chief)

Università degli Studi di Torino

Dipartimento di Matematica "Giuseppe Peano"

Via Carlo Alberto 10

10123 Torino, Italy

e-mail: susanna.teraccini@unito.it

Adolfo Ballester-Bollinches

Department d'Àlgebra

Facultat de Matemàtiques

Universitat de València

Dr. Moliner, 50

46100 Burjassot (València)

Spain

e-mail: Adolfo.Ballester@uv.es

Annalisa Buffa

IMATI - C.N.R. Pavia

Via Ferrata 1

27100 Pavia, Italy

e-mail: annalisa@imati.cnr.it

Lucia Caporaso

Dipartimento di Matematica

Università Roma Tre

Largo San Leonardo Murialdo

I-00146 Roma, Italy

e-mail: caporaso@mat.uniroma3.it

\section{Fabrizio Catanese}

Mathematisches Institut

Universitätstraße 30

95447 Bayreuth, Germany

e-mail:fabrizio.catanese@uni-bayreuth.de

Corrado De Concini

Dipartimento di Matematica

Università di Roma "La Sapienza"

Piazzale Aldo Moro 5

00185 Roma, Italy

e-mail: deconcin@mat.uniromal.it

Camillo De Lellis

Institut fuer Mathematik

Universitaet Zuerich

Winterthurerstrasse 190

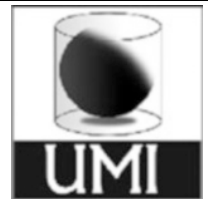

CH-8057 Zuerich, Switzerland

e-mail: camillo.delellis@math.uzh.ch

Franco Flandoli

Dipartimento di Matematica Applicata

Università di Pisa

Via Buonarroti 1c

56127 Pisa, Italy

e-mail: flandoli@dma.unipi.it

Angus Mcintyre

Queen Mary University of London

School of Mathematical Sciences

Mile End Road

London E1 4NS

United Kingdom

e-mail: a.macintyre@qmul.ac.uk

Giuseppe Mingione

Dipartimento di Matematica e Informatica

Università degli Studi di Parma

Parco Area delle Scienze, 53/a (Campus)

43124 Parma, Italy

e-mail: giuseppe.mingione@math.unipr.it

Mario Pulvirenti

Dipartimento di Matematica,

Università di Roma "La Sapienza"

P.le A. Moro 2

00185 Roma, Italy

e-mail: pulvirenti@mat.uniromal.it

Fulvio Ricci

Scuola Normale Superiore di Pisa

Piazza dei Cavalieri 7

56126 Pisa, Italy

e-mail:fricci@sns.it

Valentino Tosatti

Northwestern University

Department of Mathematics

2033 Sheridan Road

Evanston, IL 60208

USA

e-mail:tosatti@math.northwestern.edu

Corinna Ulcigrai

Forschungsinstitut für Mathematik

HG G 44.1

Rämistrasse 101

8092 Zürich, Switzerland

e-mail: corinna.ulcigrai@bristol.ac.uk

The Editorial Policy can be found at the back of the volume. 
Pascal Cherrier • Albert Milani

\section{Evolution Equations of von Karman Type}

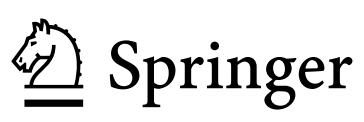


Pascal Cherrier

Départment de Mathématiques

Université Pierre et Marie Curie

Paris, France

\author{
Albert Milani \\ Department of Mathematics \\ University of Wisconsin \\ Milwaukee, WI, USA
}

ISSN 1862-9113

ISSN 1862-9121 (electronic)

Lecture Notes of the Unione Matematica Italiana

ISBN 978-3-319-20996-8

ISBN 978-3-319-20997-5 (eBook)

DOI 10.1007/978-3-319-20997-5

Library of Congress Control Number: 2015952855

Mathematics Subject Classification (2010): 53D05, 53D12, 37J05, 37J10, 35F21, 58E05, 53Z05

Springer Cham Heidelberg New York Dordrecht London

(c) Springer International Publishing Switzerland 2015

This work is subject to copyright. All rights are reserved by the Publisher, whether the whole or part of the material is concerned, specifically the rights of translation, reprinting, reuse of illustrations, recitation, broadcasting, reproduction on microfilms or in any other physical way, and transmission or information storage and retrieval, electronic adaptation, computer software, or by similar or dissimilar methodology now known or hereafter developed.

The use of general descriptive names, registered names, trademarks, service marks, etc. in this publication does not imply, even in the absence of a specific statement, that such names are exempt from the relevant protective laws and regulations and therefore free for general use.

The publisher, the authors and the editors are safe to assume that the advice and information in this book are believed to be true and accurate at the date of publication. Neither the publisher nor the authors or the editors give a warranty, express or implied, with respect to the material contained herein or for any errors or omissions that may have been made.

Printed on acid-free paper

Springer International Publishing AG Switzerland is part of Springer Science+Business Media (www.springer.com) 
To our wives, Annick and Claudia 
Tentasse Iuvat 


\section{Preface}

In these notes, we consider two kinds of nonlinear evolution problems of von Karman type on $\mathbb{R}^{2 m}, m \geq 2$. Each of these problems consists of a system that results from the coupling of two highly nonlinear partial differential equations, one hyperbolic or parabolic, and the other elliptic. These systems are called "of von Karman type" because of a formal analogy with the well-known equations of the same name in the theory of elasticity in $\mathbb{R}^{2}$.

\section{The Classical Equations}

1. To describe the classical hyperbolic von Karman system in $\mathbb{R}^{2}$, we introduce the nonlinear coupling of the second order derivatives of two sufficiently smooth functions $g=g(x, y)$ and $h=h(x, y)$, defined by

$$
[g, h]:=\operatorname{det}\left(\begin{array}{ll}
g_{x x} & g_{x y} \\
h_{y x} & h_{y y}
\end{array}\right),
$$

and then we set

$$
N(g, h):=[g, h]+[h, g]=g_{x x} h_{y y}+g_{y y} h_{x x}-2 g_{x y} h_{x y} .
$$

The classical von Karman equations in $\mathbb{R}^{2}$ consist of the system

$$
\begin{aligned}
u_{t t}+\Delta^{2} u & =N(f, u)+N(\varphi, u), \\
\Delta^{2} f & =-N(u, u),
\end{aligned}
$$

where $\Delta$ the usual Laplace operator in $\mathbb{R}^{2}$, and $\varphi=\varphi(t, x, y)$ is a given external source. Equations (3) and (4) model the dynamics of the vertical oscillations (buckling) of an elastic two-dimensional thin plate, represented by a bounded 
domain $\Omega \subset \mathbb{R}^{2}$, due to both internal and external stresses. More precisely, in this model the unknown function $u=u(t, x, y)$ is a measure of the vertical displacement of the plate; Eq. (4) formally defines a map $u \mapsto f(u)$, where $f(u)$ represents the so-called Airy stress function, which is related to the internal elastic forces acting on the plate, and depends on its deformation $u$; finally, $\varphi$ represents the action of the external stress forces. Typically, Eqs. (3)+(4) are supplemented by the initial conditions

$$
u(0)=u_{0}, \quad u_{t}(0)=u_{1},
$$

where $u_{0}$ and $u_{1}$ are a given initial configuration of the displacement and its velocity, and by appropriate constraints on $u$ at the boundary of $\Omega$.

2. A detailed and precise description of the modeling issues related to the classical von Karman equations, and a discussion of their physical motivations, can be found in, e.g., Ciarlet and Rabier [12], or in Ciarlet [10, 11]; in addition, we refer to the recent, exhaustive study by Chuesov and Lasiecka [9] of a large class of initialboundary value problems of von Karman type on domains of $\mathbb{R}^{2}$, with a multitude of different boundary conditions, including nonlinear ones. The stationary state of the classical von Karman equations, described by the nonlinear elliptic system

$$
\begin{aligned}
\Delta^{2} u & =N(f, u)+N(\varphi, u), \\
\Delta^{2} f & =-N(u, u),
\end{aligned}
$$

has been investigated by several authors; in particular, Berger [3], devised a remarkable variational method to establish the existence of suitably regular solutions to the stationary system (6)+(7) in a bounded domain of $\mathbb{R}^{2}$, subject to appropriate boundary conditions. Weak solutions of the corresponding system of evolution $(3)+(4)+(5)$, again under appropriate boundary conditions, have been established, among others, by Lions [21, Chap. 1, Sect. 4], and Favini et al. [15, 16], and Chuesov and Lasiecka [9].

\section{The Generalized Equations}

1. To introduce the generalization of the von Karman system (3)+(4) we wish to consider, we now let $m \in \mathbb{N}_{\geq 2}$, and, given $m+1$ smooth functions $u_{1}, \ldots, u_{m}, u$ defined on $\mathbb{R}^{2 m}$, we set

$$
\begin{aligned}
N\left(u_{1}, \ldots, u_{m}\right) & :=\delta_{j_{1} \cdots j_{m}}^{i_{1} \cdots i_{m}} \nabla_{i_{1}}^{j_{1}} u_{1} \ldots \nabla_{i_{m}}^{j_{m}} u_{m}, \\
M(u) & :=N(u, \ldots, u)=m ! \sigma_{m}\left(\nabla^{2} u\right),
\end{aligned}
$$


where we adopt the usual summation convention for repeated indices, and use the following notations. For $i_{1}, \ldots, i_{m}, j_{1}, \ldots, j_{m} \in\{1, \ldots 2 m\}, \delta_{j_{1} \cdots j_{m}}^{i_{1} \cdots i_{m}}$ denotes the Kronecker tensor; for $1 \leq i, j \leq 2 m, \nabla_{i}^{j}:=\partial_{i} \partial_{j}$, and $\sigma_{m}$ is the $m$-th elementary symmetric function of the eigenvalues $\lambda_{k}=\lambda_{k}\left(\partial_{i} \partial_{j} u\right), 1 \leq k \leq 2 m$, of the Hessian matrix $H(u):=\left[\partial_{i} \partial_{j} u\right]$, that is,

$$
\sigma_{m}\left(\nabla^{2} u\right):=\sum_{1 \leq k_{1}<k_{2}<\ldots<k_{m} \leq 2 m} \lambda_{k_{1}} \cdots \lambda_{k_{m}} .
$$

We also introduce the convention

$$
N\left(u_{1}^{\left(k_{1}\right)}, \ldots, u_{p}^{\left(k_{p}\right)}\right):=N(\underbrace{u_{1}, \ldots, u_{1}}_{k_{1} \text { factors }}, \ldots \underbrace{u_{p}, \ldots, u_{p}}_{k_{p} \text { factors }}),
$$

with $k_{1}+\cdots+k_{p}=m$, and set $\Delta:=-\nabla_{j}^{j} u$.

In Lemma 1.3.1 of Chap. 1, we shall show that the elliptic equation

$$
\Delta^{m} f=-M(u)
$$

can be uniquely solved, in a suitable functional frame, for $f$ in terms of $u$, thereby defining a map $u \mapsto f:=f(u)$. Let $T>0$. Given a source term $\varphi$ defined on $[0, T] \times \mathbb{R}^{2 m}$, we consider the Cauchy problem, of hyperbolic type, in which we wish to determine a function $u$ on $[0, T] \times \mathbb{R}^{2 m}$, satisfying the equation

$$
u_{t t}+\Delta^{m} u=N\left(f(u), u^{(m-1)}\right)+N\left(\varphi^{(m-1)}, u\right)
$$

and subject to the initial conditions (5), where, now, $u_{0}$ and $u_{1}$ are given functions defined on $\mathbb{R}^{2 m}$. We refer to this Cauchy problem, that is, explicitly, to $(13)+(12)+$ (5), as "problem (VKH)".

Problem (VKH) appears to be analogous to the original von Karman system (3) and (4) on $\mathbb{R}^{2}$, but this analogy is only formal, in the following sense. Let $d$ denote the space dimension. In the linear part at the left side of Eqs. (3) and (4) of the original system, the order of the differential operator $\Delta^{2}$ is twice the dimension of space (i.e., $4=2 d, d=2$ ), and the nonlinear operators of Monge-Ampère type at the right side of the equations are defined in terms of the complete Hessian of functions depending on $u, f$, and $\varphi$. In contrast, at the left side of Eqs. (13) and (12) the order of the differential operator $\Delta^{m}$ equals the dimension of space (i.e., $2 m=d$ ), while the Monge-Ampère operators at the right side of these equations are defined in terms of elementary symmetric functions of order $m=\frac{d}{2}$ of Hessian matrices of functions depending on $u, f$, and $\varphi$. To illustrate this difference explicitly, in the original equation (4) the term $N(u, u)$ is twice the determinant of the Hessian 
matrix

$$
H(u)=\left(\begin{array}{ll}
u_{x x} & u_{x y} \\
u_{y x} & u_{y y}
\end{array}\right)
$$

of $u$; since this matrix has two eigenvalues $\lambda_{1}\left(\partial_{i} \partial_{j} u\right)$ and $\lambda_{2}\left(\partial_{i} \partial_{j} u\right)$, whose product equals the determinant $u_{x x} u_{y y}-u_{x y}$ of $H(u)$, we obtain that

$$
N(u, u)=2 \operatorname{det} H(u)=2 \lambda_{1}\left(\partial_{i} \partial_{j} u\right) \lambda_{2}\left(\partial_{i} \partial_{j} u\right) .
$$

In contrast, when $m=1$ the condition $1 \leq j_{1} \leq 2 m=2$ in the sum of (10) reduces to $j_{1}=1$ or $j_{1}=2$, so that definitions (9) and (10) yield a completely different expression for $N(u, u)$, namely

$$
N(u, u)=2 ! \sigma_{1}\left(\nabla^{2} u\right)=2\left(\lambda_{1}\left(\partial_{i} \partial_{j} u\right)+\lambda_{2}\left(\partial_{i} \partial_{j} u\right)\right)
$$

The difference between (15) and (16) shows that, indeed, the analogy between the original von Karman system and the equations we consider here is only formal. For completeness' sake, we mention that the extension of the original von Karman equations (3) and (4) to even space dimension $d=2 m$ would consist of the system

$$
\begin{aligned}
u_{t t}+\Delta^{m+1} u & =N\left(f(u), u^{(2 m-1)}\right)+N\left(\varphi^{(2 m-1)}, u\right), \\
\Delta^{m+1} f & =-N(\underbrace{u, \ldots, u}_{2 m \text { factors }}),
\end{aligned}
$$

where now, instead of (8),

$$
N\left(u_{1}, \ldots, u_{d}\right):=\delta_{j_{1} \cdots j_{d}}^{i_{1} \cdots i_{d}} \nabla_{i_{1}}^{j_{1}} u_{1} \cdots \nabla_{i_{d}}^{j_{d}} u_{d} .
$$

Even though we do not consider system $(17)+(18)$ in these notes, we point out that, from an analytical point of view, its study turns out to be much simpler than that of $(13)+(12)$.

2. Our main emphasis in these notes is on the hyperbolic version of the generalized von Karman equations in $\mathbb{R}^{2 m}$, for which we have a rather complete well-posedness theory for solutions with different types of regularity, from weak to smooth; however, we shall also briefly consider the parabolic version of these equations, for which, in contrast, we only have a well-posedness theory for strong solutions. In this system, Eq. (13) is replaced by its parabolic counterpart

$$
u_{t}+\Delta^{m} u=N\left(f(u), u^{(m-1)}\right)+N\left(\varphi^{(m-1)}, u\right),
$$

with $f(u)$ still defined by (12), and $u$ is subject to the single initial condition

$$
u(0)=u_{0}
$$


We refer to the Cauchy problem (20) + (12) + (21) as "problem (VKP)".

3. We started our investigation of the generalized von Karman equations in [4], where we considered an elliptic system formally similar to $(6)+(7)$ on a compact Kähler manifold, with boundary, and arbitrary complex dimension $m \geq 2$. This generalization involved a number of analytic difficulties, due to the rather drastic role played by the limit case of the Sobolev imbedding theorem. We then considered, in [7], the corresponding hyperbolic evolution problem, and gave some partial results on the so-called strong solutions (see Definition 1.4.1 of Chap. 1) of these equations, again on a compact Kähler manifold of arbitrary complex dimension $m \geq 2$ (this explains in part why we only consider an even number $2 m$ of real variables). In $[5,6]$, we also gave some qualitatively similar results on strong solutions to the parabolic problem (VKP) on compact Kähler manifolds. Most of these results on strong solutions for both problems (VKH) and (VKP) have been extended to the whole space case (i.e., on all of $\mathbb{R}^{2 m}$ ) in the last chapter of our textbook [8], where we presented these results as an application of a general theory of quasi-linear evolution equations of hyperbolic and parabolic type. In these works, we were able to establish the existence and uniqueness of strong solutions in a suitable function space framework, by applying the linearization and fixed-point technique developed by Kato and others (see, e.g., Kato [18, 19]). Evolution systems of von Karman type can also be studied in the context of Riemannian manifolds with boundary, with a number of extra difficulties due to the curvature of the metric of the manifold, and the presence of boundary conditions.

\section{Overview of Results}

1. Our first and main goal in these notes is to present a comprehensive study of the initial value problem for the generalized model of the hyperbolic equations of von Karman type (13) $+(12)$, in the whole space $\mathbb{R}^{2 m}$, with arbitrary integer $m \geq 2$. We seek solutions to problem (VKH) with different degrees of smoothness in the space variables, as described by the index $k$ in the chain of anisotropic Sobolev spaces

$$
\mathcal{X}_{m, k}(T):=C\left([0, T] ; H^{m+k}\right) \cap C^{1}\left([0, T] ; H^{k}\right),
$$

where for $r \in \mathbb{N}, H^{r}$ is the usual Sobolev space on $\mathbb{R}^{2 m}$ (that is, $H^{r}=W^{r, 2}\left(\mathbb{R}^{2 m}\right)$ ). We obtain different results, depending on whether $k=0$ or $k>0$. If $k=0$, we are able to establish the existence of solutions in a space $\mathcal{Y}_{m, 0}(T)$ which is larger than (22); more precisely, such that

$$
\begin{aligned}
\mathcal{X}_{m, 0}(T) & \subseteq \mathcal{Y}_{m, 0}(T) \\
& \subseteq\left\{u \in L^{\infty}\left(0, T ; H^{m}\right) \mid u_{t} \in L^{\infty}\left(0, T ; L^{2}\right)\right\} ;
\end{aligned}
$$


(see (1.131) of Chap. 1). These solutions are called WEAK, and are defined globally in time; that is, for all values of $t$ in the same interval $[0, T]$ where the given source $\varphi$ is defined. In contrast, when $k>0$ we can establish the existence of solutions that are defined only on a smaller interval $[0, \tau] \subseteq[0, T]$; that is, solutions in $\mathcal{X}_{m, k}(\tau)$, for some $\tau \in] 0, T]$. We call these STRONG, LOCAL solutions. Remarkably, the value of $\tau$ is independent of $k$; in fact, it only depends, in a generally decreasing fashion, on the size of the data $u_{0}$ in $H^{m+1}, u_{1}$ in $H^{1}$, and $\varphi$ in the space $S_{m, 1}(T)$ defined in (1.137) of Chap. 1. In addition, these strong solutions depend continuously on the data $u_{0}, u_{1}$, and $\varphi$, in a sense described precisely at the end of Chap. 1 .

2. A similar kind of results holds for the initial value problem for the generalized model of the parabolic equations of von Karman type (20) + (12), again in the whole space $\mathbb{R}^{2 m}, m \geq 2$. Here too, we seek solutions to problem (VKP) with different degrees of smoothness in the space variables, described by the index $h$ in the chain of isotropic Sobolev spaces

$$
\mathcal{P}_{m, h}(T):=\left\{u \in L^{2}\left(0, T ; H^{m+h}\right) \mid u_{t} \in L^{2}\left(0, T ; H^{h-m}\right)\right\} .
$$

When $h \geq m$, these solutions are called STRONG, and as in the hyperbolic case we are able to establish the existence of strong, local solutions in $\mathcal{P}_{m, h}(\tau)$, for some $\tau \in$ ]0,T]. Again, $\tau$ is independent of $h$, and its size depends, in a generally decreasing fashion, on the size of the data $u_{0}$ in $H^{m}$ and $\varphi$ in the space $S_{m, 0}(T)$ defined in (1.137) of Chap.1. In addition, these strong solutions depend continuously on the data $u_{0}$ and $\varphi$, in a sense described precisely at the end of Chap. 1 . Weak solutions correspond to the case $0 \leq h<m$ in (24); however, in contrast to the hyperbolic case, we are not able to even give a meaningful definition of weak solutions to problem (VKP) in the context of the spaces $\mathcal{P}_{m, k}(T)$, except when $m=2$.

3. These notes are organized as follows. In Chap. 1 we prepare the analytic and functional space framework in which we study the hyperbolic equations of von Karman type (3) + (4), and state the results we seek to establish. In Chap.2, we prove the existence of global weak solutions to problem (VKH), extending the above-cited result of Lions [21], to arbitrary even space dimension $2 m$. In Chap. 3, we prove the local well-posedness of the equations in a suitable strong sense, when $m+k \geq 4$, and in Chap. 4, we prove a weaker well-posedness result for the exceptional case $m=2, k=1$. In Chap. 5, we briefly consider the parabolic version $(20)+(12)$ of the von Karman equations, and establish a result on the local existence and uniqueness of strong solutions of problem (VKP), and one on the existence of weak solutions when $m=2$. In contrast to our earlier work (as summarized, e.g., in [8, Chap. 7, Sect. 2]), all existence results here are established via suitable Galerkin approximation schemes. Finally, in Chap.6, we report some technical results on the Hardy space $\mathcal{H}^{1}$ on $\mathbb{R}^{N}$, which we then use to show the well-posedness of weak solutions of problem (VKH) for the classical von Karman equations (3) + (4) in $\mathbb{R}^{2}$.

4. While the physical significance of the von Karman system we consider may not be evident, the interest of this problem resides chiefly in a number of specific analytical features, which make the study of these equations a rich subject of investigation. The 
two major difficulties we encounter are the lack of compactness, which characterizes the study of evolution equations in the whole space (and which is, obviously, not present in the case of a compact manifold, or other types of bounded domains with appropriate boundary conditions), and a lack of regularity of the second order space derivatives of the function $\partial_{x}^{2} f$ defined by (12). This difficulty is related to the limit case of the Sobolev imbedding theorem. More precisely, we encounter a drastic difference between the situation where the derivatives $\partial_{x}^{2} f(t, \cdot)$ are in $L^{\infty}$, or not. Interestingly enough, in the hyperbolic case that is of most interest to us it turns out that we are not able to determine whether this condition holds or not, only when either $m \geq 2$ and $k=0$ (case of the weak solutions), or when $m=2$ and $k=1$, which is a somewhat exceptional case; in all others, including the case $m=1, k \geq 0$ of the classical von Karman equations, the condition $\partial_{x}^{2} f(t, \cdot) \in L^{\infty}$ does hold.

\section{Acknowledgements}

We are grateful to Professors A. Negro of the Università di Torino, H. Beirão da Veiga of the Università di Pisa, and P. Secchi of the Università di Brescia for many useful discussions and valuable encouragement.

Paris, France

San Francisco, CA, USA

Pascal Cherrier

Albert Milani 



\section{Contents}

1 Operators, Spaces, and Main Results .......................... 1

1.1 Functional Framework ....................................... 1

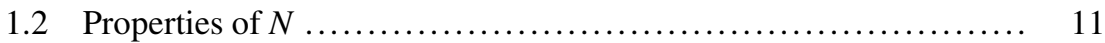

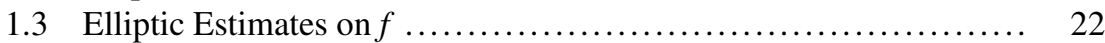

1.4 Statement of Results .................................... 25

1.5 Friedrichs' Mollifiers ...................................... 33

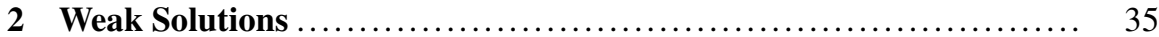

2.1 Existence of Weak Solutions ................................ 36

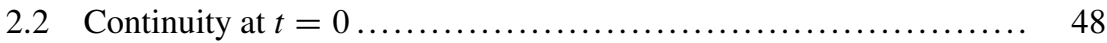

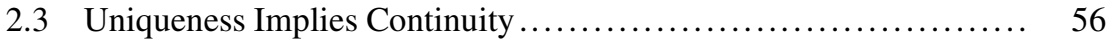

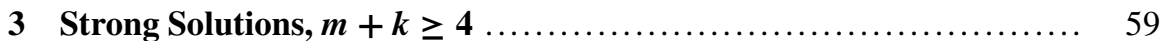

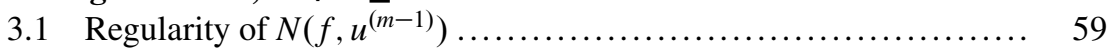

3.2 Well-Posedness ........................................... 66

3.3 Existence .............................................. 72

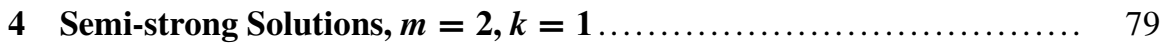

4.1 Two Technical Lemmas.......................................... 79

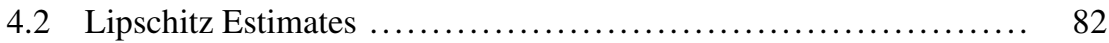

4.3 Well-Posedness ........................................ 86

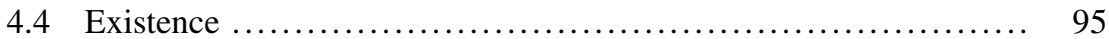

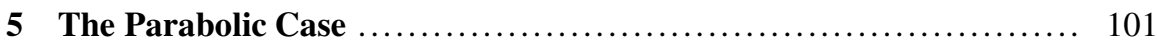

$5.1 \quad$ Well-Posedness ......................................... 101

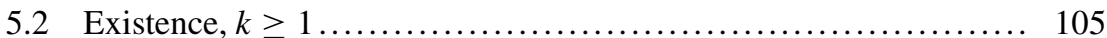

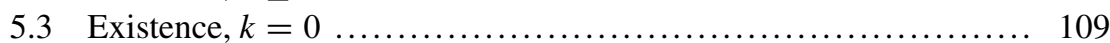

5.4 Weak Solutions ........................................ 116

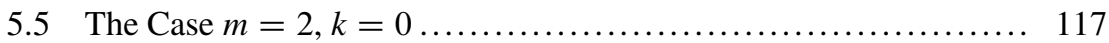




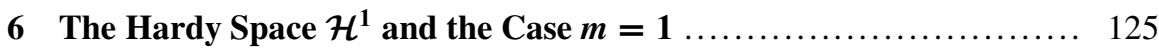

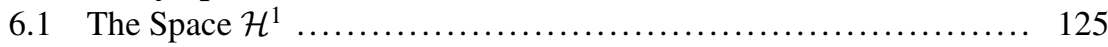

6.2 The Classical von Karman Equations ...................... 133

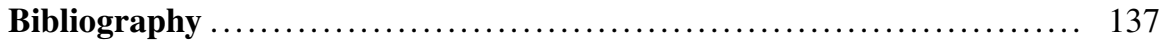

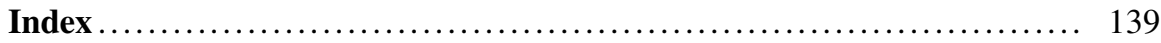

